\title{
CONTINUOUS PROPER HOLOMORPHIC MAPS INTO BOUNDED DOMAINS
}

\author{
AVNER DOR \\ (Communicated by Clifford Earle, Jr.)
}

\begin{abstract}
A continuous proper holomorphic map is constructed from the unit ball of $\mathbb{C}^{N}$ to a smooth bounded domain in $\mathbb{C}^{M}(2 \leq N \leq M-1)$. The construction is done at a strongly convex corner of the target domain. At each stage the map is pushed farther into the boundary in a direction almost tangent to the boundary at a close vicinity. The close point property is employed, along with suitable peak functions, to obtain a minimal codimension. It appears to be close to the most general construction that can be made by summation of peak functions.
\end{abstract}

Theorem 1. Let $2 \leq N \leq M-1$, and let $D \subset \subset \mathbb{C}^{M}$ be a bounded domain with $C^{2}$ boundary. Then there exists a proper holomorphic map from $B^{N}$ to $D$ which is continuous on $\bar{B}^{N}$.

The construction of this map evolved from the construction of nonsmooth proper holomorphic maps from $B^{N}$ to $B^{N+1}$ in [D1], which uses ideas from the constructions in [HS, L1, L2, F, S]. However, in [D1] the constructed proper map can approximate a given holomorphic map in the following sense: If $f: B^{N} \rightarrow B^{M}$ (where $2 \leq N \leq M-1$ ) is holomorphic, $\varepsilon>0$, and $K \subset B^{N}$ is compact, then there exists a proper holomorphic map $F: B^{N} \rightarrow B^{M}$ continuous on $\bar{B}^{N}$ such that $|F-f|<\varepsilon$ on $K$. This is not the case when the target domain is an arbitrary bounded smooth domain. A recent paper by Forstnerič and Globevnik [FG] provides an example of a bounded smooth domain $D$ in $\mathbb{C}^{M}(2 \leq M)$, with disconnected boundary, where no proper holomorphic map from $\Delta$ to $D$ goes through a prescribed point $w_{0} \in D$. Their proof can easily be adapted to show that the proper holomorphic maps from $B^{N}$ (for any $N \leq M-1)$ to the domain $D$ cannot approximate the constant map $f(z)=w_{0}$ in the above sense.

The following question remains open: Is there a proper holomorphic map from a ball (or, equivalently, a smooth, bounded, strongly pseudoconvex domain) into a general domain $D$ of higher dimension? It seems likely that the answer for the most general domain is negative, but it may be rather hard to

Received by the editors August 22, 1991 and, in revised form, March 20, 1992; these results were presented in lectures given in the Several Complex Variables Special Year at the University of Michigan, Ann Arbor, February, 1993. I thank J. E. Fornaess for the kind invitation.

1991 Mathematics Subject Classification. Primary 32H35. 
characterize the domains for which the answer is positive. Such a characterization will have to be in terms of a thin and local subset of the boundary of the target domain. Our proof gives a positive answer to this question whenever there exists $p \in b D$ with a neighborhood $G$ of $p$ such that $b D \cap G$ is $C^{2}$ smooth and $p$ is a point of strong convexity. By application of the Narasimhan Lemma (see $[R, 15.5 .3$, p. 320]) this holds also when $p$ is a point of strong pseudoconvexity. The map we constructed here lies within a small neighborhood of the point $p$.

An interesting characterization is that of target domains with connected smooth boundaries that admit approximations by proper holomorphic maps (as described above) from lower-dimensional balls. The codimension is an important parameter in these questions.

One can combine our proof with the proof of Theorem 2 in [D1] to obtain a map in Theorem 1 with an image that contains an open subset of the boundary of $D$. On the other hand, we cannot verify that the images of the maps constructed in this paper or in [D1, D2] do not contain an open subset of the boundary of the target domain in the one-codimensional case.

In [D2] a proper holomorphic map was constructed from a ball in $\mathbb{C}^{N}$ to a polydisc in $\mathbb{C}^{M}(2 \leq N \leq M-1)$; however, it was shown there that such a map cannot be continuous on any open subset of the boundary.

All the constructions of proper holomorphic maps mentioned above can be made from smooth $\left(C^{\infty}\right)$ bounded strongly pseudoconvex domains since they depend only on the existence of peak functions of the type constructed in [D1]. In [S] it is realized that such peak functions exist for strongly pseudoconvex domains with smooth boundary.

Familiarity with the proofs in [D1] is required in order to understand the proof of Lemma 1.

\section{Proof of TheOREM 1}

I. Let $p \in b D$ be such that $|p|=\max \{|z|: z \in \bar{D}\}$. Then $|p| B^{M} \supset D$. The point $p$ will be fixed for the entire proof, and we will assume that $|p|=\frac{1}{2}$. We can find $10^{-10}>\delta_{0}>0$ small enough such that to every $z \in\left(p+\delta_{0} B^{M}\right) \cap \bar{D}$ a unique point $z^{\prime} \in\left(p+2 \delta_{0} B^{M}\right) \cap b D$ with $\left|z-z^{\prime}\right|=d(z, b D)$ can be assigned. Define, for every point $z \in\left(p+\delta_{0} B^{M}\right) \cap \bar{D}, \mathbf{N}(z)$ to be the unit outer normal to the boundary of $D$ at the point $z^{\prime}$. We can assume that $\delta_{0}>0$ is small enough so that $\mathbf{N}$ is continuous on $\left(p+\delta_{0} B^{M}\right) \cap \bar{D}$ and that for every $z \in\left(p+2 \delta_{0} B^{M}\right) \cap$ $b D$ there is a ball $B$ with radius 1 containing $D$ so that $\{z\}=b D \cap b B$, and a ball $B^{\prime}$ with radius $10 \delta_{0}$ contained in $D$, where $\{z\}=b D \cap b B^{\prime}$.

II. Instead of proving Theorem 1 we will prove the following stronger theorem that implies Theorem 1.

Theorem 1*. There exists $\alpha>0$ such that if $f: b B^{N} \rightarrow D \cap\left(p+\alpha B^{M}\right)$ is continuous and $\varepsilon>0$, then there exists $g: \bar{B}^{N} \rightarrow \mathbb{C}^{M}$ continuous and holomorphic in $B^{N}$ such that $(f+g)\left(\bar{B}^{N}\right) \subset\left(p+\delta_{0} B^{M}\right) \cap \bar{D},(f+g)\left(b B^{N}\right) \subset b D$, and $|g(z)|<\varepsilon$ whenever $z \in(1-\varepsilon) \bar{B}^{N}$.

The following lemma is the main tool in the proof of Theorem $1^{*}$. After the lemma is stated, Theorem $1^{*}$ is proved by inductive application of this lemma. In the last section of the paper the technical and difficult proof of Lemma 1 is presented. 
The constant $\beta>0$ is defined in Lemma 3 of [D1] and $\varepsilon_{0} \stackrel{\text { def }}{=} 10^{-(10 N) ! ! / \beta \delta_{0}}$.

Lemma 1. For a given $z_{0} \in b B^{N}$ there exists $W$, an open neighborhood of $z_{0}$ in the topology of $\bar{B}^{N}$, such that the following holds.

For every continuous $f: b B^{N} \rightarrow D \cap B\left(p, \delta_{0}\right)$ and $\varepsilon_{0}>\varepsilon>0$ there exists $g: \bar{B}^{N} \rightarrow \mathbb{C}^{M}$ continuous and holomorphic $B^{N}$ with the following properties:

(a) for all $z \in b B^{N}, 0<d\left((f+g)(z), D^{c}\right)<\varepsilon+d\left(f(z), D^{c}\right)$;

(b) for all $z \in W \cap b B^{N}, d((f+g)(z), b D)<d(f(z), b D)\left(1-\left(\varepsilon_{0}\right)^{2}\right)$;

(c) for all $z \in b B^{N},|g(z)|^{2}<\left(\varepsilon_{0}\right)^{1 / 2} d(f(z), b D)$; and

(d) for all $z \in(1-\varepsilon) \bar{B}^{N},|g(z)|<\varepsilon$.

Lemma 1 is used to push the map increasingly toward the boundary. It will be shown by (a) and (b) that the distance to the boundary at the $n$th stage is bounded from above by $C^{n}$, where $1>C>0$ is a constant. Therefore, it will follow from (c) that the convergence is uniform and it goes to a holomorphic map that takes boundary to boundary.

1.1. Let $W_{1}, \ldots, W_{m}$ be open subsets of $\bar{B}^{N}$, where $\bigcup\left\{W_{i}: 1 \leq i \leq m\right\} \supset$ $b B^{N}$ and $W_{i} \quad(1 \leq i \leq m)$ has the properties of $W$ in Lemma 1. Assume that $m \geq 100$. The integer $m$ and the sets $W_{1}, \ldots, W_{m}$ will be fixed henceforth.

1.2. When $n$ is an integer define $\bar{n}$ to be the unique integer so that $1 \leq \bar{n} \leq m$ and $(n-\bar{n}) / m$ is an integer.

1.3. Define $\alpha=\left(\delta_{0}\right)^{2} \cdot\left(\varepsilon_{0}\right)^{20}(2 m)^{-2}$.

1.4. Define $f_{1}=f, g_{0} \equiv 0$. Let $n \geq 1$, and assume inductively that the maps $g_{0}, \ldots, g_{n-1}, f_{1}, \ldots, f_{n}$ are defined, where $f_{i}: \bar{B}^{N} \rightarrow D \cap B\left(p, \delta_{0}\right)$, $g_{j}: \bar{B}^{N} \rightarrow \mathbb{C}^{M}(1 \leq i \leq n, 0 \leq j \leq n-1)$ are continuous and holomorphic in $B^{N}$ and $f_{n}=f_{1}+g_{1}+\cdots+g_{n-1}$.

1.5. Define $\varepsilon_{n}=\varepsilon \cdot\left(\varepsilon_{0}\right)^{10} \cdot \min \left\{\left(d\left(f_{i}(z), b D\right)\right)^{2}: z \in b B^{N}, 0 \leq i \leq n\right\} / 2^{n}$, where $\varepsilon>0$ is as in the statement of Theorem $1^{*}$.

1.6. By Lemma 1 there exists a continuous map $g_{n}: \bar{B}^{N} \rightarrow \mathbb{C}^{M}$ holomorphic in $B^{N}$ so that the following hold:

(a) for all $z \in b B^{N}, 0<d\left(\left(f_{n}+g_{n}\right)(z), D^{c}\right)<\varepsilon_{n}+d\left(f_{n}(z), D^{c}\right)$;

(b) for all $z \in W_{\bar{n}} \cap b B^{N}, d\left(\left(f_{n}+g_{n}\right)(z), b D\right)<d\left(f_{n}(z), b D\right)\left(1-\left(\varepsilon_{0}\right)^{2}\right)$;

(c) for all $z \in b B^{N},\left|g_{n}(z)\right|^{2}<\left(\varepsilon_{0}\right)^{1 / 2}\left(f_{n}(z), b D\right)$; and

(d) for all $z \in\left(1-\varepsilon_{n}\right) \bar{B}^{N},\left|g_{n}(z)\right|<\varepsilon_{n}$.

Define $f_{n+1}=f_{n}+g_{n}$. The inductive process will stop at some $n \geq 1$ iff we do not have $f_{n}\left(\bar{B}^{N}\right) \subset B\left(p, \delta_{0}\right)$.

1.7. Assume now that the inductive process continues until a fixed integer $L \geq 1$.

1.8. Properties (b) and (c) imply that for all $L-1 \geq n \geq 1$ :

(1) for all $z \in b B^{N} \cap W_{\bar{n}}, d\left(f_{n}(z), b D\right)\left(1-\left(\varepsilon_{0}\right)^{2}\right)>d\left(\left(f_{n+1}\right)(z), b D\right)$; and

(2) for all $z \in b B^{N}, \varepsilon_{n}+d\left(f_{n}(z), b D\right)>d\left(\left(f_{n+1}\right)(z), b D\right)$.

When one looks at the definition of $\varepsilon_{1}, \varepsilon_{2}, \ldots$ it follows easily that for all $L-m \geq n \geq 1$

$$
d\left(f_{n}(z), b D\right)\left(1-\left(\varepsilon_{0}\right)^{3}\right)>d\left(\left(f_{n+m}\right)(z), b D\right) .
$$


1.9. We obtain from the above that for all $L \geq n>1$ and $z \in b B^{N}$

$$
2 d\left(f_{1}(z), b D\right)\left(1-\left(\varepsilon_{0}\right)^{3}\right)^{n / m}>d\left(f_{n}(z), b D\right) .
$$

1.10. Since $d\left(f_{1}(z), b D\right)<\alpha$ for all $z \in b B^{N}$, we conclude from $1.6(\mathrm{c})$ that for all $L \geq n \geq 1$

$$
\left|g_{n}\right|<\alpha^{1 / 2}\left(1-\left(\varepsilon_{0}\right)^{3}\right)^{n / 2 m} .
$$

Therefore using 1.3

$$
\begin{aligned}
\left|f_{L+1}-p\right| & \leq\left|f_{1}-p\right|+\left|g_{0}\right|+\left|g_{1}\right|+\cdots+\left|g_{L}\right| \\
& <\alpha^{1 / 2}\left(1+\left(1-\left(\varepsilon_{0}\right)^{3}\right)^{1 / 2 m}+\cdots+\left(1-\left(\varepsilon_{0}\right)^{3}\right)^{L / 2 m}\right) \\
& <\alpha^{1 / 2}\left(1-\left(1-\left(\varepsilon_{0}\right)^{3}\right)^{1 / 2 m}\right)^{-1}<\delta_{0} / 2 .
\end{aligned}
$$

Thus the process will continue until $L+1$. We have thus proven that the inductive process will not stop.

It follows from 1.10 that $\sum_{0 \leq n<\infty} g_{n}$ converges uniformly on $\bar{B}^{N}$. Call its limit $g$. Clearly $g$ is continuous on $\bar{B}^{N}$ and holomorphic on $B^{N}$.

Define $F=f+g$. Then since $F$ is a uniform limit of $\left\{f_{n}\right\}$ in $\bar{B}^{N}, F$ is continuous on $\bar{B}^{N}$ and holomorphic on $B^{N}$. By $1.9 F(z) \in b D$ whenever $z \in b B^{N}$. Thus $F$ is a proper map from $B^{N}$ to $D$. Now 1.5 and $1.6(\mathrm{~d})$ imply that $|g(z)|<\varepsilon$ whenever $z \in(1-\varepsilon) \bar{B}^{N}$, and 1.10 and $1.6(\mathrm{a})$ imply that $F\left(\bar{B}^{N}\right) \subset\left(p+\delta_{0} B^{M}\right) \cap \bar{D}$. Theorem $1^{*}$ is thus proved.

\section{Proof OF LeMMA 1}

We start with a construction which is typical for proper holomorphic maps in low codimension. We apply the definition of the peak functions in [D1] and use their properties that are developed there. Then the peak functions are distributed on the boundary of $B^{N}$ with accordance to Lemma 3 of [D1], following the presentation there. In an attempt to keep the text short, we will not repeat calculations that were done in [D1].

2.1. Assume (as we may) that $\varepsilon<\left(\varepsilon_{0} \cdot d\left(f\left(b B^{N}\right), b D\right)\right)^{100}$.

2.2. Take $r>0$ so that:

(i) $\varepsilon^{30}>r$;

(ii) when $z, w \in \bar{B}^{N}$ and $|z-w|<r^{1 / 30}$

$$
|\mathbf{N}(f(z))-\mathbf{N}(f(w))|,|f(z)-f(w)|<\varepsilon^{10} ;
$$

(iii) $(\log \varepsilon) /\left(r^{1 / 2} \cdot N^{5} \cdot 2 \pi\right)$ is an integer.

We might have to shrink $r>0$ later.

2.3. Now adopt from [D1] Definitions 0.1-0.5 and Sublemma 1 and also the definitions and calculations done in 6.1-6.10 where the $r$ is the one chosen above. At this point it is advised that the reader become familiar with this material before continuing. Define $V^{*}=\left\{X \in V^{\prime}: d(X, V)<r^{1 / 3}\right\}$.

2.4. Define for $a \in L$ and $z \in \mathbb{C}^{N}$

$$
p_{a}(z)=\exp \left(u_{a}(z) \cdot\left(\log \varepsilon_{0}\right) /\left(r N^{5}\right)\right) .
$$

So $1>\left|p_{a}\right|>0$ on $\bar{B}^{N} \backslash\left\{z_{a}\right\}$, and $p_{a}\left(z_{a}\right)=1$. We will suppress the distinction between $z$ and $X(z)$ when $z \in W^{\prime}$ and $X(z)$ represents the coordinates of $z$ 
defined in [D1, 0.1-0.5]. Thus $p_{a}(X) \stackrel{\text { def }}{=} p_{a}(z)$ when $z \in W^{\prime}$ and $X=X(z)$. Since $\varepsilon_{0}$ is a constant, this definition differs from the parallel ones in [D1, D2] and the calculations will have to change accordingly. Now adopt all of the material in [D1, 6.11-6.13] where $\varepsilon_{0}$ replaces $\varepsilon \quad\left(\varepsilon_{0}\right.$ is defined before the statement of Lemma 1).

2.5. Take $a, b \in L$ where $a^{\prime}=b^{\prime}$. Then

$$
p_{a}(X) \cdot \bar{p}_{b}(X)=\left|p_{a}(X) \cdot \bar{p}_{b}(X)\right| \cdot \theta_{a}(X) \cdot \bar{\theta}_{b}(X) .
$$

The proof in [D1, 1.12] can be employed to show that if $\operatorname{Re}\left(u_{a}(X) /\left(r N^{5}\right)\right)$, $\operatorname{Re}\left(u_{b}(X) /\left(r N^{5}\right)\right)<\left(d_{1}\right)^{-1}$, then

$$
\left|\theta_{a}(X)-1\right|,\left|\theta_{b}(X)-1\right|<10^{-20} .
$$

The proofs of (A)-(D), to follow, show that the rapid decline of the peak functions means that only those closest to the point $X$ have a significant size. By 2.5 the arguments of peak functions, whose peak points are in a close vicinity of a fixed point $X$ and have the same $y$-coordinates, are close to a constant (see definition [D1, 6.4]), and therefore such peak functions can be added without cancelling each other. Placing of the peak functions along the $y$-coordinates is done with great care, using the close point lemma in [D1, 5.1, 5.2], so that their sum does not cancel.

2.6. Let $v_{1}, v_{2}, \ldots, v_{N}: V^{*} \rightarrow \mathbb{C}^{M}$ be continuous functions, such that $\left|v_{i}(X)\right|$ $=1$ (for all $1 \leq i \leq N$ ) and $\left\{v_{1}(X), \ldots, v_{N}(x)\right\}$ are mutually orthogonal for every $X \in V^{*}$, and they are perpendicular to $\mathbf{N}(f(X))$. Such functions exist since $\mathbf{N}$ is continuous in $B\left(p, \delta_{0}\right) \cap D$, the function $f$ is continuous, and the dimension $M$ is larger than $N$. By shrinking $r$ further we can assume that when $X, Y \in V^{*}$ are such that $|X-Y|<r^{1 / 30}$ then for all $i=1,2, \ldots, N$

$$
|\mathbf{N}(f(X))-\mathbf{N}(f(Y))|,|f(X)-f(Y)|,\left|v_{i}(X)-v_{i}(Y)\right|<\varepsilon^{10} .
$$

Define for $a \in L$

$$
t_{a}=\left(\varepsilon_{0} d\left(f\left(X_{a}\right), b D\right)\right)^{1 / 2} .
$$

Take $a \in L$; then there exists a unique $i \in\{1, \ldots, N\}$ so that $a^{\prime} \in S_{i}$ (see [D1, 6.7]). Define $v_{a}=t_{a} \cdot v_{i}\left(X_{a}\right)$. One can easily verify (as in [D1, 1.14; D2, 2.25]) the following (i)-(v). For all $a, b \in L$

(i) $\quad\left(v_{a}, \mathbf{N}\left(f\left(X_{a}\right)\right)\right)=0$; and

(ii) $\quad\left|v_{a}\right|^{2}=\varepsilon_{0} d\left(f\left(X_{a}\right), b D\right)$.

Let $a, b \in L,|a-b|<r^{-0.1}$ so that $a^{\prime} \in S_{i}, b^{\prime} \in S_{j}$. Then the following hold:

(iii) if $i \neq j,\left|\left(v_{a}, v_{b}\right)\right|<\varepsilon^{5}$;

(iv) if $i=j,\left.\left|\left(v_{a}, v_{b}\right)-\right| v_{a}\right|^{2} \mid<\varepsilon^{5}$;

(v) $\left.|| v_{b}\right|^{2}-\left|v_{a}\right|^{2} \mid<\varepsilon^{5}$.

We will proceed with a few technical definitions.

2.7. Define for $z \in W^{\prime}$ and $n \geq 0$

$$
L(z, n)=\left\{a \in L: n^{2} \leq\left|\operatorname{Re}\left(u_{a}(z /|z|)\right)\right|^{2} / r N^{5}<(n+1)^{2}\right\} .
$$


2.8. When looking at the peak function distribution defined in [D1, 6.5-6.8], we obtain the following estimates for all $z \in W^{\prime}, n \geq 0$ :

(1) $\operatorname{car}\left(\bigcup_{0 \leq k \leq n} L(z, k)\right)<\left(C_{N}\right) \cdot(n+1)^{2 N}$, where $C_{N}$ is a positive constant that depends on the dimension $N, C_{N}<N^{N}$ (we will now fix $C_{N}$ ).

(2) When $a \in L(z, n),\left|p_{a}(z)\right| \leq\left(\varepsilon_{0}\right)^{n^{2} / 2+\left(1-|z|^{2}\right) / 2 r N^{5}}$.

(3) When $z \in z(V)($ see $[\mathrm{D} 1,0.3]) L(z, 0) \neq \varnothing$.

2.9. As in [D1] when $z \in W^{\prime}$ is fixed we will define for $a \in L[a]=n$, where $n$ is the only integer so that $a \in L(z, n)$.

2.10. Define for $z \in \bar{B}^{N}$ and $1 \leq i \leq N \quad h_{i}(z)=\sum_{a \in L_{i}} p_{a}(z) \cdot v_{a}$ and $h=h_{1}+h_{2}+\cdots+h_{N}$ (see [D1, 6.7-6.9] and 2.4, 2.6 above). We will identify $h(X)$ (where $X \in U^{\prime}$ ) with $h(z(X))$.

2.11. The following is true for the map $h$ :

(A) For all $z \in W^{\prime},|(\mathbf{N}(f(z)), h(z))|<\varepsilon^{3}$.

(B) For all $z \in W^{\prime},|h(z)|^{2}<\frac{1}{2}\left(\varepsilon_{0}\right)^{\left(1 / 2+\left(1-|z|^{2}\right) /\left(r N^{5}\right)\right)} \cdot d(f(z), b D)$.

(C) For all $z \in W \cap b B^{N},|h(z)|^{2}>10\left(\varepsilon_{0}\right)^{2} d(f(z), b D)$.

(D) If $z \in W^{\prime}$ and $d(z, z(V))>r^{0.1}$ then $|h(z)|^{2}<\varepsilon^{100}$.

Similar lemmas appear in all constructions of proper holomorphic maps from strongly pseudoconvex domains.

2.12. Fix $X=\left(X_{1}, \ldots, X_{2 N-1}, 0\right) \in V$, and let $t_{j}=X_{j} / c_{j}(j=1, \ldots, 2 N-$ 1) and $t=\left(t_{1}, \ldots, t_{2 N-1}\right)$. Let || denote the standard Euclidean norm. By Lemma 3 in [D1] there exists $i \in\{1, \ldots, N\}$ and $a \in L_{i}$ so that $\left|t^{\prime}-\hat{a}^{\prime}\right|+\beta<$ $\left|t^{\prime}-\hat{b}^{\prime}\right|$ for every $b \in L_{i}$ such that $a^{\prime} \neq b^{\prime}$ (see definition [D1,6.6]). Take the smallest such $i$ and call it $i(X)$.

2.13. Choose $a(X) \in L_{i(X)}$ so that

$$
\operatorname{Re}\left(u_{a(X)}(X)-R_{a(X)}(X)\right)=\min \left\{\operatorname{Re}\left(u_{a}(X)-R_{a}(X)\right): a \in L_{i(X)}\right\}
$$

(the choice may not be unique).

2.14. It follows from the calculations in 6.21-6.27 of [D1] (when we put $\varepsilon=\varepsilon_{0}$ there) that $\left|p_{a(X)}(X)\right|>\left(\varepsilon_{0}\right)^{1 / 3}$, and if $a \in L_{i(X)}$ where $a^{\prime} \neq a^{\prime}(X)$ then

$$
\left|p_{a}(X) / p_{a(X)}(X)\right|<\left(\varepsilon_{0}\right)^{\left(\beta N^{-10}\right)} .
$$

This means, as our next calculations will show, that for every point $X \in V$ the peak function $p_{a(X)}$ dominates $\left\{p_{a}: a \in L_{i(X)}\right\}$ in the sense that in the sum $\sum_{a \in L_{i(X)}} p_{a}(X) v_{a}$ only $p_{a(X)}(X) v_{a(X)}$ is significant and the rest of the terms sum up to a small proportion of it. This is the important part in the proof of (C) and a major step in the whole proof.

The notation $a(z) \stackrel{\text { def }}{=} a(X(z)), l(r) \stackrel{\text { def }}{=}-\log (r), f\left(X_{a}\right) \stackrel{\text { def }}{=} f\left(z_{a}\right)$, and $L(X, n) \stackrel{\text { def }}{=} L(z(X), n)$ will be used in the following calculations. 
Proof of (B) and (D) (with the use of 2.8).

$$
\begin{aligned}
|h(z)|^{2}= & \left|\sum_{a, b \in L}\left(v_{a}, v_{b}\right) p_{a}(z) \bar{p}_{b}(z)\right| \\
\leq & \sum_{0 \leq m \leq l(r)} \sum_{a, b \in L,[a]+[b]=m}\left|\left(v_{a}, v_{b}\right) p_{a}(z) \bar{p}_{b}(z)\right| \\
& +\sum_{l(r)<m} \sum_{0, b \in L,[a]+[b]=m}\left|p_{a}(z) \bar{p}_{b}(z)\right| \\
< & \sum_{0 \leq m \leq l(r)}\left(\left|v_{a(z)}\right|^{2}+\varepsilon^{4}\right) \cdot\left(C_{N}\right)^{2}(m+1)^{4 N} \cdot\left(\varepsilon_{0}\right)^{m^{2} / 4+\left(1-|z|^{2}\right) / r N^{5}} \\
& +\sum_{l(r)<m}\left(C_{N}\right)^{2}(m+1)^{4 N} \cdot\left(\varepsilon_{0}\right)^{m^{2} / 4+\left(1-|z|^{2}\right) / r N^{5}} \\
< & \left(\left(C_{N}\right)^{3}\left(\left|v_{a(z)}\right|^{2}+\varepsilon^{4}\right)+\left(\varepsilon_{0}\right)^{l(r)}\right) \cdot\left(\varepsilon_{0}\right)^{\left(1-|z|^{2}\right) / r N^{5}} \\
< & \left(\left(C_{N}\right)^{3} \cdot\left(\varepsilon_{0}\right) \cdot d(f(z), b D)+\varepsilon^{3}\right) \cdot\left(\varepsilon_{0}\right)^{\left(1-|z|^{2}\right) / r N^{5}} \\
< & \frac{1}{2}\left(\varepsilon_{0}\right)^{1 / 2} d(f(z), b D) \cdot\left(\varepsilon_{0}\right)^{\left(1-|z|^{2}\right) / r N^{5}} .
\end{aligned}
$$

We used the size of $\varepsilon>0$ at 2.1 .

In the case that $z \notin z\left(V^{*}\right)$ we can take $a(z)$ to be any fixed element in $\bigcup_{0 \leq n \leq l(r)} L(z, n)$. If this set is empty then the calculation above is trivial since the sum $\sum_{0 \leq m \leq l(r)} \cdots=0$, and we obtain that $|h(z)|^{2}<\left(\varepsilon_{0}\right)^{l(r)}<$ $\varepsilon^{100}$. This certainly happens whenever $d(z /|z|, z(V))>r^{0.2}$. In the case that $d(z, z(V))>r^{0.1}$ then we have $d(z /|z|, z(V))>r^{0.2}$ or $1-|z|^{2}>r^{0.2}$. In either case it follows from the calculation above that $|h(z)|^{2}<\varepsilon^{100}$; thus, (D) is also proved.

$\operatorname{Proof}$ of (A). By (B) we may assume that $|z|>1-r^{1 / 2}$ (if not then $|h(z)|<\varepsilon^{100}$, and (A) follows). When $a \in L(z, n)$ and $n \leq l(r)$ we have $\left|z-z_{a}\right|<r^{1 / 3}$ and by $2.2\left|\mathbf{N}(f(z))-\mathbf{N}\left(f\left(z_{a}\right)\right)\right|<\varepsilon^{10}$. Using also $2.6(\mathrm{i})$ by which for all $a \in L$ $\left(v_{a}, \mathbf{N}\left(f\left(X_{a}\right)\right)\right)=0$, we then have

$$
\begin{aligned}
|(\mathbf{N}(f(z)), h(z))|=\left|\sum_{a \in L}\left(\mathbf{N}(f(z)), v_{a}\right) \bar{p}_{a}(z)\right| & \left|\mathbf{N}(f(z))-\mathbf{N}\left(f\left(z_{a}\right)\right)\right| \cdot\left|v_{a}\right|\left|p_{a}(z)\right| \\
\leq & \sum_{n \leq l(r)} \sum_{a \in L(z, n)}\left|p_{a}(z)\right|\left|v_{a}\right| \\
& +\sum_{l(r)<n} \sum_{a \in L(z, n)} 2 C_{N} \cdot(n+1)^{2 N} \varepsilon^{10} \cdot\left(\varepsilon_{0}\right)^{n^{2} / 2} \\
< & \sum_{0 \leq n \leq l(r)} 2 C^{2 N} \cdot\left(\varepsilon_{0}\right)^{n^{2} / 2}<\varepsilon^{3} \\
& +\sum_{l(r)<n} 2 C_{N} \cdot(n+1)^{2}
\end{aligned}
$$

Note that when $L(z, n)$ is empty, the above calculations trivially hold.

Proof of $(\mathrm{C})$. We will follow the structure of the proof of $(\mathrm{C})$ in [D1]. Fix $X \in V$ (recall that $\left.V=X\left(W \cap b B^{N}\right)\right)$. 
2.15. When $1 \leq i, j \leq N$ and $i \neq j$ then:

Proof.

$$
\left|\left(h_{i}(X), h_{j}(X)\right)\right|<\varepsilon^{3} \text {. }
$$

$$
\begin{aligned}
\left|\left(h_{i}(X), h_{j}(X)\right)\right|= & \sum_{0 \leq m \leq l(r)} \sum_{a \in L_{i}, b \in L_{j},[a]+[b]=m}\left(v_{a}, v_{b}\right) p_{a}(X) \bar{p}_{b}(X) \\
& +\sum_{l(r)<m} \sum_{a \in L_{i}, b \in L_{j},[a]+[b]=m}\left(v_{a}, v_{b}\right) p_{a}(X) \bar{p}_{b}(X) \mid \\
& <\sum_{0 \leq m \leq l(r)} \varepsilon^{5}\left(C_{N}\right)^{2}(m+1)^{4 N} \cdot\left(\varepsilon_{0}\right)^{m^{2} / 4} \\
& +\sum_{l(r)<m}\left(C_{N}\right)^{2}(m+1)^{4 N} \cdot\left(\varepsilon_{0}\right)^{m^{2} / 4}<\varepsilon^{3} .
\end{aligned}
$$

2.16. Proposition. $\left|h_{i(X)}(X)\right|^{2}>\left(\varepsilon_{0}\right)^{1.5} d(f(X), b D)-\varepsilon^{3}$.

Propositions 2.15 and 2.16 imply (C) since $h=h_{1}+\cdots+h_{N}$ and because of the choice of $\varepsilon$ in 2.1 .

2.17. Define $A(X)=\left\{a \in L_{i(X)}:[a] \leq 100, a^{\prime}=a^{\prime}(X)\right\}$ and $B(X)=$ $L_{i(X)} \backslash A(X)$. Then

$$
\left|h_{i(X)}(X)\right|^{2} \geq\left|\sum_{a \in A(X)} p_{a}(X) v_{a}\right|^{2}-2\left|\sum_{a \in A(X), b \in B(X)}\left(v_{a}, v_{b}\right) p_{a}(X) \bar{p}_{b}(X)\right| .
$$

Note that

$$
\left|\sum_{a \in A(X)} p_{a}(X) v_{a}\right|^{2}=\sum_{a, b \in A(X)} \operatorname{Re}\left(\left(v_{a}, v_{b}\right) p_{a}(X) \bar{p}_{b}(X)\right) .
$$

When $a, b \in A(X)$, since $a^{\prime}=b^{\prime}$ and $[a],[b] \leq 100$, by $2.5 \operatorname{Re}\left(p_{a}(X) \bar{p}_{b}(X)\right)$ $>0$, and by 2.6(iv), (v), since $a, b \in L_{i(X)}$, then $\left.\left|\left(v_{a}, v_{b}\right)-\right| v_{a(X)}\right|^{2} \mid<\varepsilon^{4}$; therefore; $\operatorname{Re}\left(\left(v_{a}, v_{b}\right) p_{a}(X) \bar{p}_{b}(X)\right)>-\varepsilon^{4}$. Since $a(X) \in A(X)$ and $\operatorname{car}(A(X)) \leq$ $\sum_{0 \leq n \leq 100} C_{N}(n+1)^{2 N}<10^{8 N} \cdot N^{N} \stackrel{\text { def }}{=} M_{0}$, we obtain

$$
\left|\sum_{a \in A(X)} p_{a}(X) v_{a}\right|^{2}>\left|v_{a(X)}\right|^{2}\left|p_{a(X)}(X)\right|^{2}-\varepsilon^{3.5}
$$

Recall that this result was hinted at in the remark following 2.5. Let $b \in B(X)$, $[b]=0$. Then since $b^{\prime} \neq a^{\prime}(X)$, it follows from 2.14 that

$$
\left|p_{b}(X) / p_{a(X)}(X)\right|<\left(\varepsilon_{0}\right)^{\beta N^{-10}} .
$$

When $b \in B(X)$ and $[b]=n \geq 1$, by $2.8(2)\left|p_{b}(X)\right|<\left(\varepsilon_{0}\right)^{n^{2} / 2}$, and since $\left|p_{a(X)}(X)\right|>\left(\varepsilon_{0}\right)^{1 / 3}$, we have that

$$
\left|p_{b}(X) / p_{a(X)}(X)\right|<\left(\varepsilon_{0}\right)^{n^{2} / 2-1 / 3} .
$$


2.19. It follows that when $b \in B(X),[b]=n \geq 0$

$$
\left|p_{b}(X) / p_{a(X)}(X)\right|<\left(\varepsilon_{0}\right)^{n^{2} / 8+\beta N^{-10}} .
$$

(2.20)

$$
\begin{aligned}
\mid & \left.\sum_{a \in A(X), b \in B(X)} \mid v_{a}, v_{b}\right) p_{a}(X) \bar{p}_{b}(X) \mid \\
\leq & \sum_{\substack{a \in A(X) \\
b \in B(X),[b] \leq l(r)}}\left|\left(v_{a}, v_{b}\right) p_{a}(X) \bar{p}_{b}(X)\right| \\
& +\sum_{\substack{a \in A(X) \\
b \in B(X),[b]>l(r)}}\left|\left(v_{a}, v_{b}\right) p_{a}(X) \bar{p}_{b}(X)\right| \\
& <\sum_{\substack{a \in A(X) \\
b \in B(X),[b] \leq l(r)}}\left(\left|v_{a(X)}\right|^{2}+\varepsilon^{4}\right) \cdot\left|p_{a}(X)\right|\left|\bar{p}_{b}(X)\right|+\sum_{\substack{a \in A(X) \\
b \in B(X),[b]>l(r)}}\left|\bar{p}_{b}(X)\right| \\
& <\sum_{0 \leq n}\left(\left(\left|v_{a(X)}\right|^{2}+\varepsilon^{4}\right) \cdot\left|p_{a(X)}(X)\right|^{2}\right) M_{0} C_{N}(n+1)^{2 N} \cdot\left(\varepsilon_{0}\right)^{\left(n^{2} / 8+\left(\beta N^{-10}\right)\right)} \\
& +M_{0} \sum_{\substack{l(r)<n \\
\left(\varepsilon_{0}\right)^{n^{2} / 2}}}^{<} \\
< & \left(\left|v_{a(X)}\right|^{2}+\varepsilon^{4}\right) \cdot\left|p_{a(X)}(X)\right|^{2} \cdot\left(\varepsilon_{0}\right)^{\beta N^{-20}}+\left(\varepsilon_{0}\right)^{l(r)} \\
< & \left|v_{a(X)}\right|^{2} \cdot\left|p_{a(X)}(X)\right|^{2} \cdot\left(\varepsilon_{0}\right)^{\beta N^{-20}}+\varepsilon^{3.5} .
\end{aligned}
$$

From this and $\left|p_{a(X)}(X)\right|>\left(\varepsilon_{0}\right)^{1 / 3}$ of $(2.14)$ we obtain (considering as usual the size of $\varepsilon>0)$ :

$$
\begin{aligned}
\left|h_{i(X)}(X)\right|^{2} & >\left|v_{a(X)}\right|^{2}\left|p_{a(X)}(X)\right|^{2} \cdot\left(1-2\left(\varepsilon_{0}\right)^{\beta N^{-20}}\right)-3 \varepsilon^{3.5} \\
& >\frac{1}{2} \varepsilon_{0} \cdot\left|p_{a(X)}(X)\right|^{2} \cdot d(f(X), b D)-\varepsilon^{3} \\
& >\left(\varepsilon_{0}\right)^{1.7} \cdot d(f(X), b D)-\varepsilon^{3}>10\left(\varepsilon_{0}\right)^{2} \cdot d(f(X), b D)
\end{aligned}
$$

(C) is now proved.

2.22. Applying the globalization process of [D1, 1.34-1.38] we obtain from (D), after possibly shrinking $r>0$ in the definition of $h$, that there exists a $C^{\infty}$ map $g: \bar{B}^{N} \rightarrow \mathbb{C}^{M}$ which is holomorphic in $B^{N}$ such that:

(i) for all $z \in W^{\prime},|g(z)-h(z)| z<\varepsilon^{40}$; and

(ii) for all $z \in \bar{B}^{N} \backslash W^{\prime} ;|g(z)|<\varepsilon^{40}$.

2.23. When $z \in \bar{B}^{N} \backslash W^{\prime}$ then since $|g(z)|<\varepsilon^{40}, 2.1$ implies that Lemma 1 clearly holds in this case. Now fix (until 2.27) $z \in W^{\prime}$. By I (at the beginning of the proof of Theorem 1) there exists a ball $B \subset \mathbb{C}^{M}$, with radius 1 so that $\left\{f(z)^{\prime}\right\}=b B \cap b D$ and $D \subset B$, and a ball $B^{\prime} \subset \mathbb{C}^{M}$, with radius $10 \delta_{0}$ so that $\left\{f(z)^{\prime}\right\}=b B^{\prime} \cap b D$ and $B^{\prime} \subset D$. Note that $f(z)^{\prime}-f(z)$ is a real scalar product $\mathbf{N}(f(z))$. Let $q$ be the center of the ball $B^{\prime}$. Then since $\mathbf{N}(f(z))$ is also the normal of $b B^{\prime}$ at $f(z)^{\prime}$, it follows that $f(z)-q$ is also a real scalar product of $\mathbf{N}(f(z))$ and $\left\{f(z)^{\prime}, f(z), q\right\}$ lie in one real line. Observe that $\left|f(z)-f(z)^{\prime}\right|=d(f(z), b D)$. 
2.24. Now $|(f(z)-q, g(z))| \leq|(\mathbf{N}(f(z)), g(z))|$, and by (A) and 2.22 $|(\mathbf{N}(f(z)), g(z))|<\varepsilon^{2}$ and also by (B) and $2.22|g(z)|^{2}<\left(\varepsilon_{0}\right)^{1 / 2}\left|f(z)-f(z)^{\prime}\right|$. We then get (using the size of $\varepsilon>0$ in 2.1 and the size of $\varepsilon_{0}$ ) that

$$
\begin{aligned}
& |(f(z)+g(z))-q|^{2} \leq|f(z)-q|^{2}+2|(f(z)-q, g(z))|+|g(z)|^{2} \\
& \quad<|f(z)-q|^{2}+2 \varepsilon^{2}+\left(\varepsilon_{0}\right)^{1 / 2}\left|f(z)-f(z)^{\prime}\right| \\
& \quad=\left(10 \delta_{0}-\left|f(z)-f(z)^{\prime}\right|\right)^{2}+2 \varepsilon^{2}+\left(\varepsilon_{0}\right)^{1 / 2}\left|f(z)-f(z)^{\prime}\right|<\left(10 \delta_{0}\right)^{2} .
\end{aligned}
$$

Hence $f(z)+g(z) \in B^{\prime} \subset D$.

2.25. We continue with a similar reasoning with respect to the ball $B$. Let $w$ be the center of the ball $B$. Then $\left\{w, f(z), f(z)^{\prime}\right\}$ lie on one real line and $d(f(z), b B)=\left|f(z)-f(z)^{\prime}\right|=d(f(z), b D)$. Since $f(z)-w$ is a real scalar product of $\mathbf{N}(f(z))$ and $|f(z)-w|<1$, it follows from (A) and 2.22 that $|(f(z)-w, g(z))| \leq|(\mathbf{N}(f(z)), g(z))|<\varepsilon^{2}$.

\subsection{Observe that}

$$
\begin{aligned}
|(f(z)+g(z))-w|^{2}-|f(z)-w|^{2} & \geq-2|(f(z)-w, g(z))|+|g(z)|^{2} \\
& >|g(z)|^{2}-2 \varepsilon^{2}
\end{aligned}
$$

and since $f(z)+g(z) \in B^{\prime} \subset B, 1>|(f(z)+g(z))-w|,|f(z)-w|>1 / 2$. Therefore

$$
\begin{aligned}
|(f(z)+g(z))-w|-|f(z)-w| & =\frac{|(f(z)+g(z))-w|^{2}-|f(z)-w|^{2}}{|(f(z)+g(z))-w|+|f(z)-w|} \\
& >\frac{1}{2}|g(z)|^{2}-2 \varepsilon^{2} .
\end{aligned}
$$

2.27. Since $D \subset B$, we obtain, using 2.26 , that

$$
\begin{aligned}
d(f(z)+g(z), b D) & \leq d(f(z)+g(z), b B)=1-|(f(z)+g(z))-w| \\
& <1-|f(z)-w|+2 \varepsilon^{2}-\frac{1}{2}|g(z)|^{2} \\
& =d(f(z), b B)+2 \varepsilon^{2}-\frac{1}{2}|g(z)|^{2} \\
& =d(f(z), b D)+2 \varepsilon^{2}-\frac{1}{2}|g(z)|^{2} .
\end{aligned}
$$

Now (a) follows from 2.24 and 2.27 and (c) from (B) and 2.22. If we assume that $z \in W \cap b B^{N}$ then (C), 2.22, and 2.27 yield (b). At last, (d) follows from (D) and 2.22. Lemma 1 is now proved.

\section{ADDED IN PROOF}

Recently the author has found an example of a bounded domain in $\mathbb{C}^{M}$, $M \geq 2$, that contains no proper images of the unit disk. Thus a smoothness assumption in Theorem 1 is necessary.

\section{REFERENCES}

[D1] A. Dor, Proper holomorphic maps between balls in one co-dimension, Ark. Mat. 28 (1990), 49-100.

[D2] _ L Lifting of proper holomorphic maps, preprint.

[F] F. Forstnerič, Embedding strictly pseudoconvex domains into balls, Trans. Amer. Math. Soc. 295 (1986), 347-368. 
[FG] F. Forstnerič and J. Globevnik, Discs in pseudoconvex domains, preprint, 1991.

[G] J. Globevnik, Relative embeddings of discs into convex domains, Invent. Math. 98 (1989), 331-350.

[H] M. Hakim, Applications propres continues de domaines strictement pseudoconvexes de $\mathbb{C}^{n}$ dans la boule unit $\mathbb{C}^{n+1}$, Duke Math J. 60 (1990), 115-133.

[HS] M. Hakim and N. Sibony, Fonction holomorphes sur la boule unite de $\mathbb{C}^{n}$, Invent. Math. 67 (1982), 213-222.

[L1] E. Løw, A construction of inner functions on the unit ball in $\mathbb{C}^{p}$, Invent. Math. 67 (1982), 223-229.

[L2] _ Embeddings and proper holomorphic maps of strictly pseudoconvex domains into polydiscs and balls, Math. Z. 190 (1985), 401-410.

[N] A. Noell and B. Stensønes, Proper holomorphic maps from weakly pseudoconvex domains, preprint.

[R] W. Rudin, Function theory in the unit ball of $\mathbb{C}^{n}$, Springer, New York, 1980.

[S] B. Stensønes, Proper holomorphic mappings from strongly pseudoconvex domains in $\mathbb{C}^{2}$ to the unit polydisc in $\mathbb{C}^{3}$, Math. Scand. 65 (1989), 129-139.

Department of Mathematics, Ben Gurion University of the Negev, 84105 Beer-Sheva, ISRAEL

E-mail address: csweinba@weizmann.weizmann.ac.il 